\section{Check for updates}

Cite this: Soft Matter, 2018, 14,2329

Received 13th November 2017, Accepted 22nd February 2018

DOI: $10.1039 / \mathrm{c} 7 \mathrm{sm} 02233 f$

rsc.li/soft-matter-journal

\title{
Defect formation dynamics in curved elastic surface crystals $\dagger$
}

\author{
Norbert Stoop and Jörn Dunkel (D) *
}

\begin{abstract}
Topological defects shape the material and transport properties of physical systems. Examples range from vortex lines in quantum superfluids, defect-mediated buckling of graphene, and grain boundaries in ferromagnets and colloidal crystals, to domain structures formed in the early universe. The KibbleZurek (KZ) mechanism describes the topological defect formation in continuous non-equilibrium phase transitions with a constant finite quench rate. Universal $K Z$ scaling laws have been verified experimentally and numerically for second-order transitions in planar Euclidean geometries, but their validity for nonthermal transitions in curved and topologically nontrivial systems still poses open questions. Here, we use recent experimentally confirmed theory to investigate topological defect formation in curved elastic surface crystals formed by stress-quenching a bilayer material. For both spherical and toroidal crystals, we find that the defect densities follow KZ-type power laws. Moreover, the nucleation sequences agree with recent experimental observations for spherical colloidal crystals. Our results suggest that curved elastic bilayers provide an experimentally accessible macroscopic system to study universal properties of non-thermal phase transitions in non-planar geometries.
\end{abstract}

\section{Introduction}

Topological defects influence the elastic, magnetic, electronic and optical properties in many natural and man-made systems. Examples range from liquid crystals ${ }^{1-4}$ to grain boundaries in ferromagnets and colloidal crystals, ${ }^{5,6}$ and charge transport in graphene $^{7,8}$ and superconductors. ${ }^{9-12}$ Topological defects can be created by varying a control parameter, such as temperature or a magnetic field, rapidly across a phase transition. Understanding the complex nonequilibrium dynamics induced by these nonadiabatic quenches remains an important theoretical challenge. Early progress in the characterization of topological defects was made by $\mathrm{Kibble}^{13}$ in 1976, while studying domain formation during the rapid cooling of the early universe. ${ }^{14}$ About a decade later, Zurek ${ }^{9}$ showed how the defect density is related to the quench rate in general second-order phase transitions; this important breakthrough also advanced significantly the understanding of topological defect formation in quantum superfluids and superconductors. ${ }^{15}$ Since then, the Kibble-Zurek (KZ) power-law scaling predictions were confirmed experimentally and utilized in a variety of systems, including liquid crystals, ${ }^{3,16}$ colloidal monolayers, ${ }^{17}$ ion crystals, ${ }^{18}$ Bose-Einstein condensates, $^{19}$ superfluids $^{10,12,20}$ and cold atomic clouds. ${ }^{21}$

Department of Mathematics, Massachusetts Institute of Technology,

77 Massachusetts Avenue, Cambridge, MA 02139-4307, USA. E-mail: dunkel@mit.edu

$\dagger$ Electronic supplementary information (ESI) available. See DOI: 10.1039/ c7sm02233f
KZ-scalings were also suggested to govern the defect formation in Rayleigh-Bénard convection, ${ }^{22-24}$ although, in contrast to theory, ${ }^{25}$ experimentally measured exponents appeared to depend on non-universal system properties. Moreover, since previous theory and experiments focused primarily on phase transitions in planar Euclidean spaces, much less is known about the existence of KZ-type scaling laws in more complex geometries and topologies.

Topological defects in curved two-dimensional (2D) crystal structures arise in many biological and physical processes, ${ }^{27,28}$ from plant growth ${ }^{29-31}$ and assembly of bacterial cell walls, ${ }^{32,33}$ viral capsids ${ }^{34,35}$ and microtubules ${ }^{36}$ to the targeted design of carbon nanotube sensors ${ }^{37}$ and microlense fabrication. ${ }^{38}$ On closed manifolds, Euler's theorem ${ }^{39}$ links the net charge of the topological defects to the genus $g$ of the underlying surface, imposing for example the 12 pentagons on a soccer ball. Depending on the details of the crystallization process (quench rate, geometric constraints, etc.), non-planar 2D crystals typically contain additional excess defects with zero total charge. ${ }^{40-42}$ Recent studies provided important experimental and theoretical insights into the defect statistics and formation dynamics in 2D colloidal crystals assembled on curved liquid-liquid interfaces. ${ }^{26,43-45}$ Another promising class of experimental systems are curved elastic bilayer systems, ${ }^{46}$ consisting of a soft substrate and a stiff surface film (Fig. 1A), which can develop hexagonal wrinkling patterns under lateral compression induced by surface swelling $^{47}$ or substrate depressurization. ${ }^{48,49}$ Such elastic surface crystals allow the realization of nontrivial shapes of genus $g>0$, 

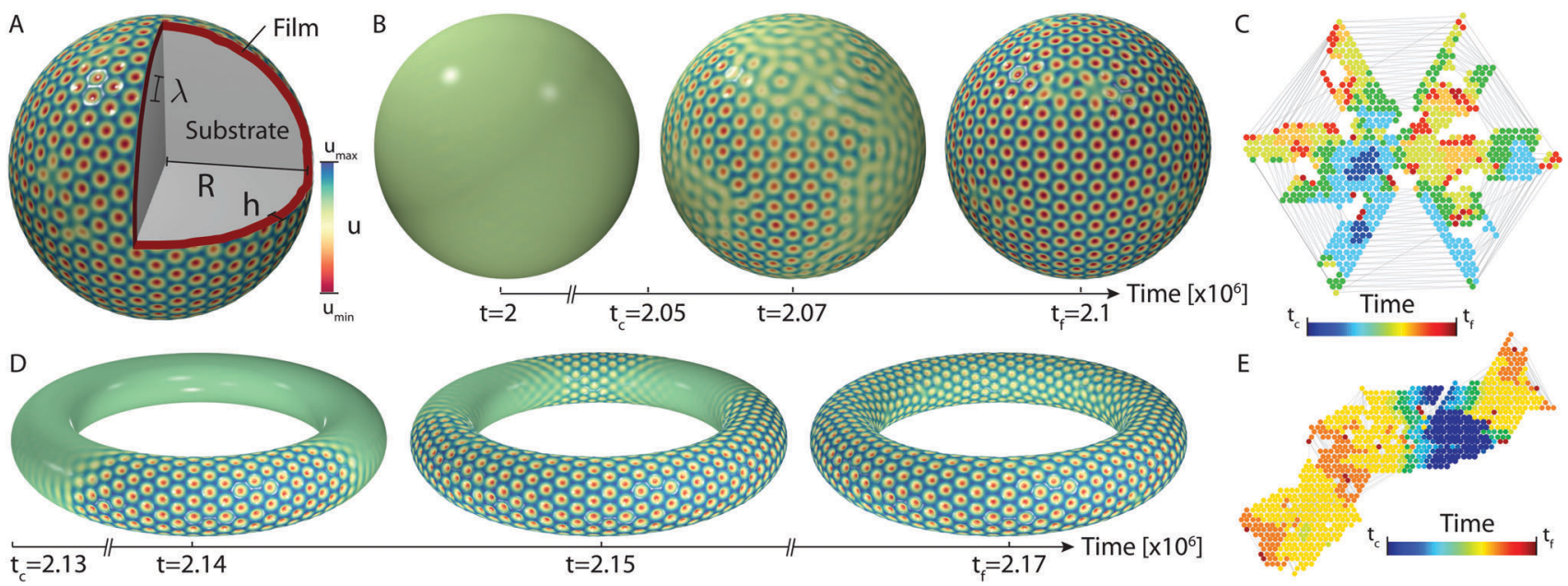

Fig. 1 Growth dynamics of elastic surface crystals in curved geometries. (A) Schematic of the simulated elastic bilayer material consisting of a thin film (thickness $h$ ) adhering to a soft substrate (radius $R$ ). Upon increasing the compressive film stress, the normal displacement $u$ of the film develops a hexagonal pattern with wavelength $\lambda$. (B) Surface crystallization dynamics on a sphere (radius $R / h=80$; Movie 1 , ESI + ) for a slow quench $\mu=5 \times 10^{-8}$. (C) Planar reconstruction of the crystallization process for the sphere in panel B. The growth of the elastic surface crystal from two initial nucleation sites (dark blue) proceeds along a predominantly regular hexagonal lattice structure, in close analogy with recent experimental observations for colloidal crystals on spherical liquid-liquid interfaces. ${ }^{26}$ (D) Crystallization dynamics on a torus (radii $R / h=120$ and $r / h=24$; Movie 2, ESI $\dagger$ ) for a slow quench $\left(\mu=5 \times 10^{-8}\right)$. (E) The planar reconstruction for the torus in panel $\mathrm{D}$ reveals that toroidal surface crystals also grow sequentially along a regular hexagonal lattice structure, centered around wave-like geodesics of minimal absolute curvature.

such as toroids, which are difficult to achieve in liquids. Moreover, because the transition from the unwrinkled to the hexagonal phase is tunable by a global control parameter, ${ }^{49}$ these soft matter systems also provide an ideal testbed to study generalizations of the $\mathrm{KZ}$ scaling laws to curved geometries.

The classical KZ argument for thermally induced secondorder transitions builds on the fact that the correlation length $\xi$ and relaxation time $\tau$ diverge as $\xi \propto \vartheta^{-\nu}$ and $\tau \propto \vartheta^{-z \nu}$ (critical slowing down), respectively, when the temperature $\vartheta$ is varied to drive the system continuously from the high-symmetry (e.g. isotropic) phase to the lower-symmetry crystal phase. The critical exponents $\nu$ and $z$ encode the universality class of the transition. For a linear quench $\vartheta=\mu t$ with rate $\mu$, the system will not be able to relax to equilibrium during the time interval $|t| \lesssim t_{\mathrm{f}}=\tau$, yielding the freeze-out condition $t_{\mathrm{f}} \propto\left(\mu t_{\mathrm{f}}\right)^{-z \nu}$ or, equivalently, $t_{\mathrm{f}} \propto \mu^{-z \nu /(1+z \nu)}$. The associated correlation length $\xi_{\mathrm{f}}=\xi\left(t_{\mathrm{f}}\right) \propto \mu^{-\nu /(1+z \nu)}$ implies the KZ scaling prediction for the defect density at freeze-out, $\rho_{\mathrm{f}} \propto \xi_{\mathrm{f}}^{-d} \propto \mu^{d \nu /(1+z \nu)}$, where $d$ is the space dimension. The analysis below shows that this argument also holds for the stress-induced pattern formation transitions observed on the 2D surfaces of curved elastic bilayer materials. Even though our system is (weakly) first-order, its dynamics arrests under sufficiently fast quenches before the coexistence region is entered, so that the classical KZ scalings can be expected to hold (see Discussion below). ${ }^{22,24}$ Furthermore, our computations identify a dynamical analogy with recent experimental observations ${ }^{26}$ on colloidal crystal formation in curved liquid-liquid interfaces. Altogether, these results suggest that pattern formation in elastic bilayers provides an experimentally promising system to study the dynamics of phase transitions in non trivial geometries. From a practical perspective, the subsequent analysis offers guidance for how to combine quench dynamics and surface geometry to control both the frequency and localization of topological defects.

\section{Theory}

To investigate topological defect formation in curved geometries, we analyze an experimentally validated continuum model $^{49}$ for the surface wrinkling in elastic bilayer materials consisting of a soft core and a stiffer outer shell (Fig. 1A). This generalized Swift-Hohenberg (GSH) theory can reproduce quantitatively the experimentally measured equilibrium phase diagrams, ${ }^{49}$ but its dynamical implications have not yet been fully explored.

\subsection{GSH theory for elastic surface crystals}

The GSH equations follow from the nonlinear Koiter shell theory ${ }^{50}$ by expanding the elastic energy of film and substrate in the dominant normal displacement field $u{ }^{49}$ Measuring length in units of the film thickness $h$, the surface energy functional of the GSH theory reads ${ }^{49}$

$$
\mathscr{E}=\frac{k}{2} \int_{\omega} \mathrm{d} \omega\left[\gamma_{0}(\nabla u)^{2}+\frac{1}{12}(\Delta u)^{2}+a u^{2}+\frac{c}{2} u^{4}-\Gamma(u)\right]
$$

where $k=E_{\mathrm{f}} /\left(1-\nu^{2}\right)$ for a film with Young's modulus $E_{\mathrm{f}}$ and Poisson ratio $\nu$, and $\mathrm{d} \omega$ is the surface element of the undeformed substrate. The nonlinear term $\Gamma(u)=\left[(1-\nu) b^{\alpha \beta} \nabla_{\alpha} u \nabla_{\beta} u+\right.$ $\left.\nu b_{\alpha}^{\alpha}(\nabla u)^{2}\right] u$ represents stretching forces to leading order in the curvature tensor $b^{\alpha \beta}$, with surface gradient $\nabla$ and LaplaceBeltrami operator $\Delta$ (throughout, Greek indices run over the set $\{1,2\}$ and Einstein's summation convention is used). Taking the variation of $\mathscr{E}$ with respect to $u$ and assuming overdamped 
dynamics, the surface wrinkling process is described by the GSH equation $^{41,49}$

$$
\tau_{0} \frac{\partial}{\partial t} u=\gamma_{0} \Delta u-\frac{1}{12} \Delta^{2} u-a u-c u^{3}-\frac{1}{2} \delta_{u} \Gamma(u)
$$

where $\gamma_{0}<0, c>0$, and $\delta_{u} \Gamma(u)$ denotes the functional derivative of the $\Gamma$-contribution to the energy functional $\mathscr{E} . \tau_{0}$ is the inverse relaxation speed; ${ }^{49}$ with no loss of generality, we set $\tau_{0}=1$ in the following. Explicit expressions ${ }^{49}$ for the coefficients and $\delta_{u} \Gamma(u)$ are summarized in the Appendix Wrinkling theory.

Linear stability analysis of eqn (1) implies that wrinkling patterns form via a discontinuous transition (see Fig. 4 in ref. 49), when the control parameter $a$ falls below the critical value $a_{\mathrm{c}}=3 \gamma_{0}{ }^{2}$, corresponding to an increase of the film stress $\sigma$ beyond the critical buckling stress $\sigma_{\mathrm{c}}$. In the following, we use the previously established definition of the excess film stress (or overstress) $\Sigma_{\mathrm{e}}=\left(\sigma / \sigma_{\mathrm{c}}\right)-1$ as control parameter. ${ }^{51}$ Here, $\sigma_{\mathrm{c}}$ is the critical film stress necessary to buckle the film from the homogeneous solution $u=0 . \sigma$ is the applied film stress, defined as the equi-biaxial equilibrium film stress in the (possibly unstable) unwrinkled solution with $u=0$. Therefore, $\sigma$ and $\Sigma_{\mathrm{e}}$, respectively, are well defined control parameters, but are not measures of the actual stress field in the deformed film. The bifurcation parameter $a$ is related to $\Sigma_{\mathrm{e}}$ by ${ }^{49}$

$$
a=a_{\mathrm{c}}-\frac{3 c}{4} \Sigma_{\mathrm{e}}
$$

In the regime beyond but still relatively close to the wrinkling threshold, $0<\Sigma_{\mathrm{e}} \sim 1$, nonlinear stability analysis confirms ${ }^{49}$ that the wrinkling solutions adopt a hexagonal pattern (Fig. 1) due to the $\delta_{u} \Gamma(u)$-term, which is breaking the $u \rightarrow-u$ symmetry of eqn (1).

\subsection{Stress-quenching of elastic surface crystals}

To obtain scaling predictions for the topological defect formation in elastic surface crystals, we first solve eqn (1) numerically for linear stress quenches

$$
\Sigma_{\mathrm{e}}(t)=\mu t
$$

with constant quench rate $\mu$, driving the system from the unwrinkled to the hexagonal phase. In all simulations, material parameters are chosen to match the experimental values reported in ref. 41 and 49 (Appendix Wrinkling theory). Essential differences compared with classical $\mathrm{KZ}$ scenarios are (i) the absence of fluctuations in eqn (1) and (ii) and that the underlying substrate geometries are non-planar. Fluctuations in thermal systems can lead to the activation of defects near the Ginzburg temperature. ${ }^{15}$ Thermal defect activation is however negligible if the temperature at freeze-out, $\vartheta\left(t_{\mathrm{f}}\right)$, is significantly lower than the Ginzburg temperature, since the system remains effectively frozen until $\vartheta\left(t_{\mathrm{f}}\right)$ is reached, and the defect density is thus determined ${ }^{15}$ by the correlation length at $t_{\mathrm{f}}$. Substrate curvature not only enters through the derivative terms, but, more importantly, determines the magnitude of the symmetrybreaking terms in eqn (1).
To break the symmetry of the initially unwrinkled surface $u=0$, a small stationary random field $\varepsilon$ with $|\varepsilon| \ll 1$ is added to the rhs of eqn (1) in simulations (Appendix Wrinkling theory). This $\varepsilon$-inhomogeneity effectively models initial imperfections in the film displacement, thus mimicking realistic experimental conditions. ${ }^{46}$ We simulate eqn (1) for the linear quench (3) and a given realization of $\varepsilon$ using the algorithm described in ref. 49. Numerical results presented below are averages over $n$ different realizations of $\varepsilon$, with $n$ specified on the corresponding graphs.

\section{Results}

To illustrate the effects of locally varying curvature and surface topology, we compare simulations for spherical and toroidal surfaces.

\subsection{Surface crystal growth under slow quenching}

For slow quasi-adiabatic quenches $(\mu \rightarrow 0)$, we find that the crystallization process is initiated at isolated nucleation sites after a critical time $t_{\mathrm{c}}$ and then spreads to cover the entire film (Fig. 1B and D). This behavior is observed for both spheres and tori (Movies 1 and 2, ESI $\dagger$ ). Details of the spreading dynamics and local crystal orientation become evident by reconstructing the corresponding planar crystal patterns as suggested by Meng et $a{ }^{26}{ }^{26}$ (Appendix Pattern analysis). Starting from a random crystal site and one of its neighbors, we determine the relative positions of all other sites connected to this initial pair, resulting in the planar crystal representations of Fig. 1C and E. Neighboring sites on the curved crystal are indicated by gray lines if they appear separated in the planar embedding. The time evolution in these graphs shows how initially separated crystal patches merge to form a single connected crystal covering the entire surface. The radial cuts appearing in Fig. $1 \mathrm{C}$ reflect the non-isometric character of the planar representation of spherical crystals, whereas the toroidal crystals unfold nearly isometrically (Fig. 1E). Moreover, crystals on the torus nucleate first near the outer rim (Fig. 1D), suggesting an influence of Gaussian curvature in the pattern-forming process in agreement with previous findings. ${ }^{52}$ As discussed below, qualitatively similar crystallization sequences were observed in a recent experiment ${ }^{26}$ that studied the deposition of charged colloids onto a spherical oil-water interface. It is important to stress that the observed crystal nucleation is a consequence of the nonlinear terms in eqn (1). Essential symmetry-breaking of the unwrinkled phase $u=0$ however already occurs at earlier times and smaller amplitudes, where these nonlinearities are negligible. Therefore, even though we observe a crystallization reminiscent of a nucleate-and-grow dynamics, the order parameter field has at this point already globally acquired a symmetry-broken phase of small amplitude (Fig. 1B, 2nd snapshot, Movie 4, ESI $\dagger$ ). This behavior is similar to the spinodalassisted nucleation of hexagonal defects. ${ }^{53,54}$ To connect with the ideas of Kibble $^{13}$ and Zurek, ${ }^{9}$ we next use the GSH elasticity model to analyze the relation between topological defect formation and quench rate $\mu$, which is difficult to explore in curved 
colloidal systems due to experimental limitations on the particle deposition rates.

\subsection{KZ-type scaling in spherical surface crystals}

We start our numerical scaling analysis by considering the case of globally constant curvature as realized in spheres (Fig. 2; Movie 1, ESI $\dagger$ ). In our simulations of eqn (1), the quench rate is varied over the range $\mu \in\left[5 \times 10^{-8}, 10^{-4}\right]$, consistent with the assumption of an overdamped dynamics. Fixing the sphere radius $R / h=80$, we characterize the transition from the unwrinkled to the wrinkled phase in terms of the average squared displacement $\left\langle u^{2}\right\rangle$, where the brackets indicate an instantaneous surface average. In the adiabatic limit $\mu \searrow 0$, this order parameter vanishes in the unwrinkled phase, $\left\langle u^{2}\right\rangle=0$ for $\Sigma_{\mathrm{e}}<0$, and jumps to a finite value $\left\langle u^{2}\right\rangle>0$ when the excess film stress $\Sigma_{\mathrm{e}}$ crosses zero from below. By contrast, for nonadiabatic quenches, we find that the system remains longer in the unwrinkled phase, before eventually breaking symmetry at some finite positive value $\Sigma_{\mathrm{e}}>0$ (Fig. 2D). Such delayed symmetry-breaking is characteristic of the classical KZ mechanism, reflecting the critical slowing down in the relaxation dynamics near a second-order phase transition. To quantify the scaling behavior in our system, we define the net freeze-out film stress $\Delta \Sigma_{\mathrm{f}}=\Sigma_{\mathrm{e}, \mathrm{f}}-\Sigma_{0}$, with $\Sigma_{\mathrm{e}, \mathrm{f}}$ being the value at which the pattern amplitude is fully developed (Appendix Freeze-out). The constant shift $\Sigma_{0} \approx 0.1$ is required to account for the material imperfections modeled by the $\varepsilon$-inhomogeneity, and is wellstudied in the context of delayed bifurcations. ${ }^{55,56}$ Our simulations confirm power-law scaling $\Delta \Sigma_{\mathrm{f}} \propto \mu^{1 / 2}$ (Fig. 2E), implying that the freeze-out time diverges as $t_{\mathrm{f}} \propto \mu^{-1 / 2}$.
The perhaps most interesting observable is the topological defect density $\rho_{\mathrm{f}}$ at freeze-out $t_{\mathrm{f}}$. Defects are crystal sites with coordination number $Z \neq 6$ and non-zero topological charge $s=6-Z$. To identify the $\mu$-dependence of $\rho_{\mathrm{f}}$ in the GSH theory, we determined the coordination number for each lattice site from the Voronoi cells of the displacement field $u$ (Appendix Pattern analysis). The resulting Voronoi graphs show that the freeze-out density $\rho_{\mathrm{f}}$ increases with the quench rate $\mu$ (Fig. 2A-C). After a quench is completed, defect pairs are expected to annihilate by grain boundary movements. We tested this hypothesis in simulations by stopping the quench at the freeze-out value, $\Sigma(t)=\Sigma_{\mathrm{e}, \mathrm{f}}$ for $t>t_{\mathrm{f}}$, so that the spherical surface crystals could relax to a stress equilibrium. For all considered quench rates, we find that the defect density approaches constant asymptotic values, which converge to the equilibrium value $\rho_{\infty}$ as $\mu \searrow 0$ (Fig. 2F). Note that, although the net topological charge is always +12 in agreement with Euler's theorem for hexagonal sphere tilings, ${ }^{39}$ charge-neutral pairs of penta- and heptagonal defects can reduce the elastic energy, ${ }^{41,44,57-59}$ resulting in a non-zero defect density $\rho_{\infty}$ even at equilibrium. Defining the relative excess defect density at freeze-out $t_{\mathrm{f}}$ by $\Delta \rho_{\mathrm{f}}=\rho_{\mathrm{f}}-\rho_{\infty}$, our numerical data is consistent with a KZ-type power law $\Delta \rho_{\mathrm{f}} \propto \mu^{1 / 2}$ (Fig. 2G).

\subsection{KZ-type scaling for toroidal surface crystals}

Local curvature variations can influence the equilibrium defect localization in curved elastic crystals. $^{41}$ Moreover, during crystal growth, local curvature profoundly affects growth rates, orientation, and defect density at impinging domains. ${ }^{52,60}$ In essence, locally varying curvature thus makes the crystallization process inhomogeneous. It is then a priori not clear if
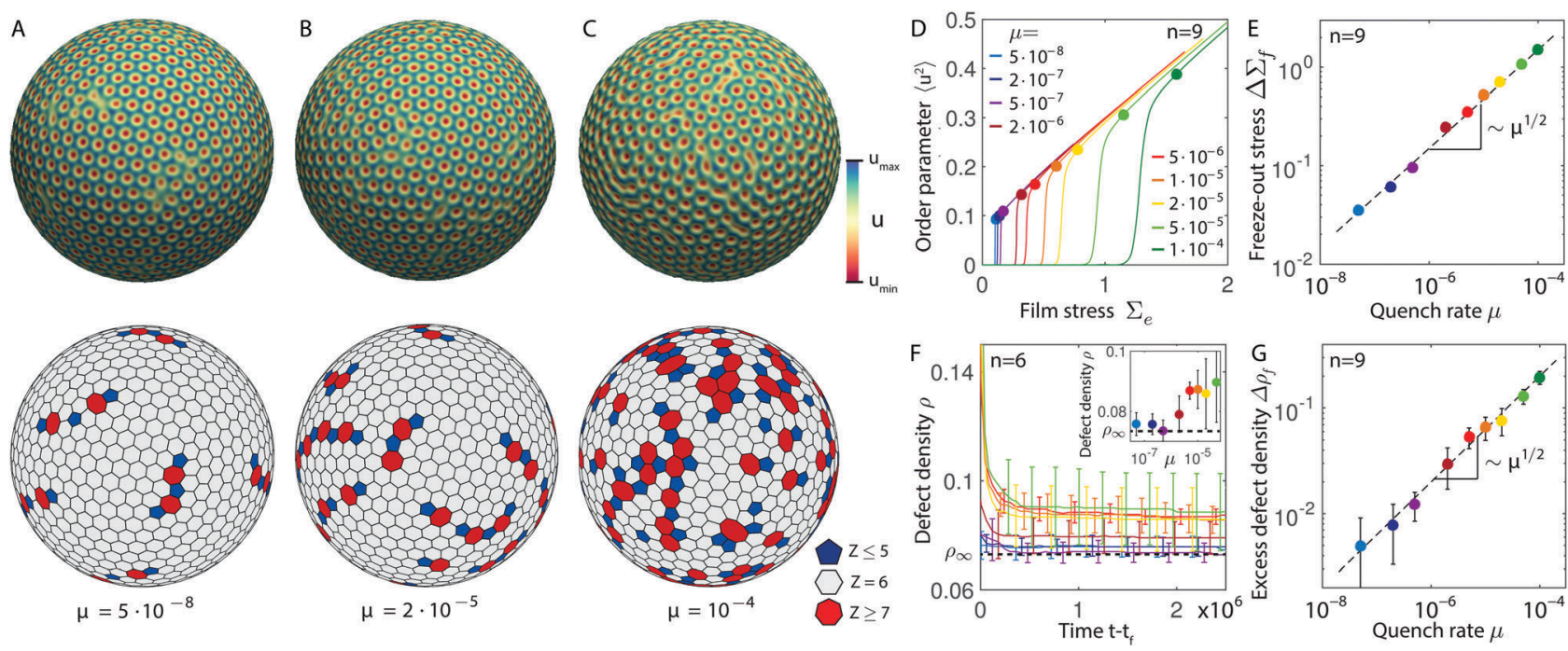

Fig. 2 KZ-type scaling laws for spherical surface crystals. (A-C) Crystalline surface patterns (top) and their corresponding Voronoi constructions (bottom) for different quench rates $\mu$ at freeze-out time $t_{\mathrm{f}}$ show an increase in the defect density for fast quenches. (D) With increasing quench rate $\mu$, bifurcation of the order parameter $\left\langle u^{2}\right\rangle$ becomes delayed, signaling non-adiabatic slowing down. Filled circles indicate the freeze-out film stress $\Sigma_{\mathrm{e}, \mathrm{f}}$ at which the system has resumed dynamics. (E) The net freeze-out stress $\Delta \Sigma_{\mathrm{f}}=\Sigma_{\mathrm{e}, \mathrm{f}}-\Sigma_{0}$ follows a power-law scaling in the quench rate consistent with classical Kibble-Zurek predictions. Error bars are smaller than symbol size. (F) When the quench is stopped at $\Sigma_{\text {e,f }}$, the system relaxes slowly to an equilibrium configuration by lowering its defect density, approaching the minimal equilibrium value $\rho_{\infty}$ in the adiabatic limit $\mu \rightarrow 0$ (inset). (G) Although the pattern formation transition is of first-order, the excess defect density $\Delta \rho_{f}=\rho_{f}-\rho_{\infty}$ at freeze-out exhibits a square-root power law scaling. Analogous results were found for systems with $R / h=40$. 

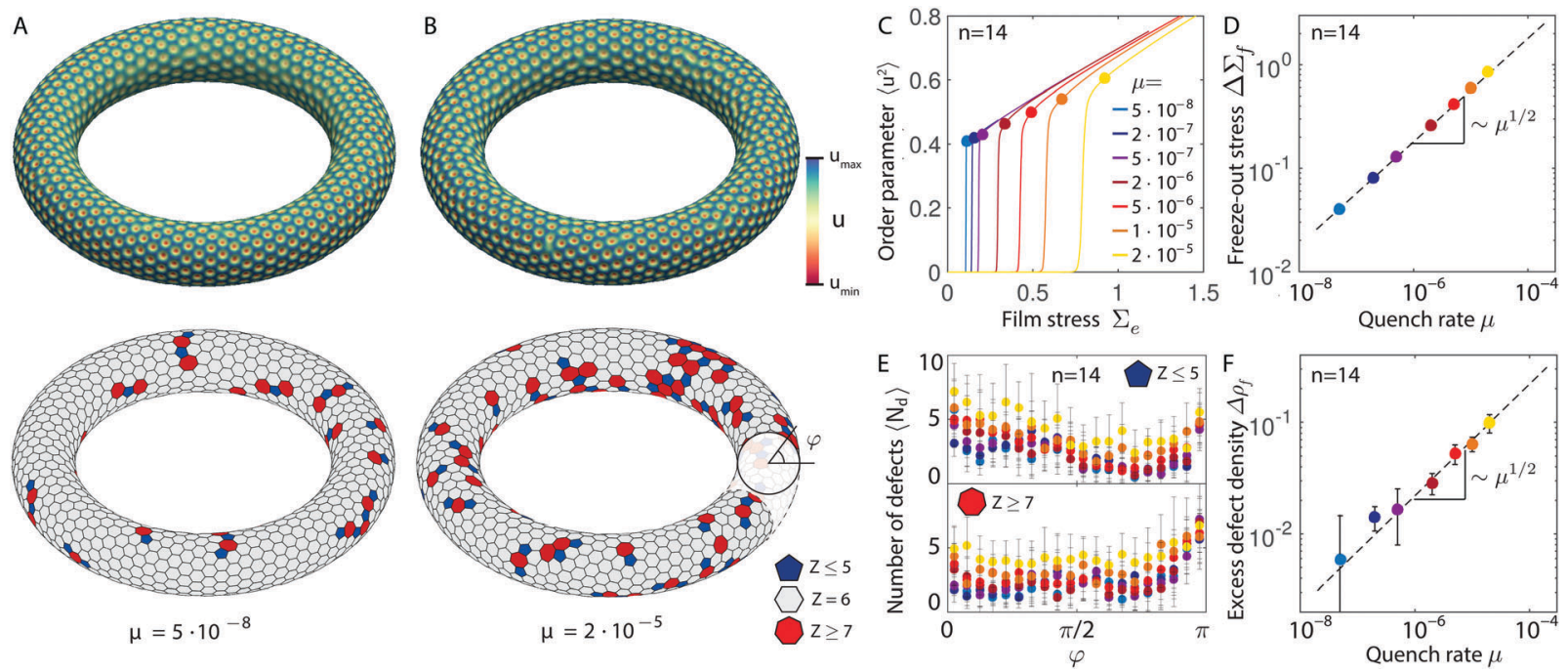

Fig. 3 KZ-type scaling laws for toroidal surface crystals. (A and B) Crystal structures (top) and Voronoi construction (bottom) for different quench rates $\mu$ at freeze-out time $t_{f}$ show an increase in the defect density for faster quenches. (C) Delayed bifurcation of the order parameter $\left\langle u^{2}\right\rangle$ for different quench rates, with the filled circles indicating the freeze-out film stress $\Sigma_{\text {e,f. }}$ (D) As for spherical substrates, we find $\Delta \Sigma_{\mathrm{f}} \propto \mu^{1 / 2}$ for tori, confirming KZ-type scaling independent of surface genus and curvature. Error bars are smaller than symbol size. (E) The local average number of defects $\left\langle N_{d}\right\rangle$ at freeze-out time $t_{f}$ depends weakly on the angle $\phi$ along the minor radius ( $\phi=0$ at the outer equator, and $\phi=\pi$ at the inner equator). (F) The locally varying curvature does not affect the scaling law for the average freeze-out excess defect density $\Delta \rho_{\mathrm{f}}=\rho_{\mathrm{f}}-\rho_{\infty}$ which exhibits a square-root dependence on $\mu$.

and under what conditions the KZ-scalings found for spherical systems also hold for geometries with non-constant curvature. To test to what degree the defect scaling laws are affected by curvature variations and surface genus, we performed additional parameter scans for tori. For elastic bilayer wrinkling, the locally varying curvature on a torus determines the strength of the symmetry-breaking term $\delta_{u} \Gamma(u)$ in eqn (1), implying that in our system purely crystalline toroidal surface patterns exist only for sufficiently small aspect ratios $r / R \cdot{ }^{41}$ We therefore focus on thin tori with $r / R=0.2$ (Fig. 3A, B and Movie 2, ESI $\dagger$ ). Adopting the same small random $\varepsilon$-inhomogeneities as for the sphere simulations (and, hence, the same shift value $\Sigma_{0}$ ), we observe that the freeze-out stress $\Delta \Sigma_{\mathrm{f}} \propto \mu^{1 / 2}$ and the freeze-out time $t_{\mathrm{f}} \propto \mu^{-1 / 2}$ scale exactly as in the case of constant curvature (Fig. $3 \mathrm{C}$ and D). Furthermore, one again finds that faster quenches lead to a higher density of defects at freeze-out (Fig. 3A, B, F and Movie 3, ESI $\dagger$ ). Although Euler's theorem ${ }^{39}$ imposes a vanishing total defect charge $S=0$ on a torus, excess defects of opposite charge tend to aggregate at the inner $(Z=7)$ and outer $(Z=5)$ equators to lower the elastic energy of the toroidal crystal, ${ }^{40,41,61,62}$ resulting in a nonzero equilibrium defect density $\rho_{\infty}$. As in the sphere case, we find that the relative excess defect density $\Delta \rho_{\mathrm{f}}=\rho_{\mathrm{f}}-\rho_{\infty}$ of the toroidal surface crystals follows a square root law $\Delta \rho_{\mathrm{f}} \propto \mu^{1 / 2}$ (Fig. 3F), suggesting that local curvature variations and surface genus do not significantly affect the scaling laws on tori. In the next part, we will rationalize these observations by considering the structure of the amplitude equations ${ }^{63}$ for the underlying GSH theory.

\section{Discussion}

As outlined in the introduction, the original $\mathrm{KZ}$ scaling relations for continuous second-order transitions can be obtained by analyzing how correlation length and relaxation time diverge as a function of the control parameter as one approaches the critical point from the high-symmetry phase. By contrast, the wrinkling transition considered here is subcritical, with a discontinuity in the order parameter at the critical stress $\Sigma_{\mathrm{e}}=0$. The transition thus carries the signature of a (weakly) firstorder transition. We note, however, that the quench starts in the unwrinkled phase $u=0$. Increasing $\Sigma_{\mathrm{e}}<0$ with stationary small perturbations $|\varepsilon| \ll 1$, the system remains in the unwrinkled phase despite the sub-critically stable hexagonal solution. Within the general Ginzburg-Landau mean-field framework, in this phase the correlation length diverges as the system approaches the critical point, and the critical exponent is $\nu=1 / 2 .{ }^{64}$ In the subcritical region, this argument is not valid due to the coexistence of solutions. The width $\delta \Sigma_{\mathrm{e}}$ of the subcritical zone is, however, small. For a spherical substrate, we previously found $\delta \Sigma_{\mathrm{e}} \approx(h / R)^{2} /\left(20 \times 0.019^{2}\right) \approx 0.02 .{ }^{49} \mathrm{As}$ pointed out in the context of Rayleigh-Bénard convection, ${ }^{22,24}$ for sufficiently fast quenches, we expect the system dynamics to freeze with a selected correlation length before the coexistence phase is entered. Considering that in our simulations, the slowest quench froze at $\left|\Delta \Sigma_{\mathrm{f}}\right| \approx 0.04>\delta \Sigma_{\mathrm{e}}$ (Fig. 1E), we are well within this regime. The critical exponent $z \nu=1$ for the divergence of the relaxation time follows by considering the characteristic time scale of the linearized decay and growth of fluctuations. ${ }^{24,64}$

\subsection{Amplitude equations}

Alternatively, we can determine the quench-dependent freeze-out time directly by inspecting the dynamics of the amplitude equations governing the GSH model. To this end, we assume approximate hexagonal solutions of the 
form $u=U(t) \sum_{a=1}^{3}\left[\mathrm{e}^{i \mathbf{k}_{a} \cdot \mathbf{x}}+\mathrm{e}^{-i \mathbf{k}_{a} \cdot \mathbf{x}}\right], \quad$ where $\quad \mathbf{k}_{1}=k_{\mathrm{c}}(1,0)$, $\mathbf{k}_{2}=k_{\mathrm{c}}(-1 / 2, \sqrt{3} / 2), \mathbf{k}_{3}=k_{\mathrm{c}}(-1 / 2,-\sqrt{3} / 2)$ and $k_{\mathrm{c}}=\sqrt{6\left|\gamma_{0}\right|}$. Inserting this ansatz into the GSH eqn (1), one finds to leading order in $U$ and curvature (Appendix Amplitude equations)

$$
\frac{\mathrm{d}}{\mathrm{d} t} U \approx \frac{c \mu t}{12 \gamma_{0}^{4}} U
$$

Dividing by $\sqrt{\mu}$ and defining a rescaled time $t^{\prime}=\mu^{1 / 2} t$, we can remove the quench rate dependence at leading order, to obtain

$$
\frac{\mathrm{d}}{\mathrm{d} t^{\prime}} U \approx \frac{c t^{\prime}}{12 \gamma_{0}{ }^{4}} U .
$$

Identifying the characteristic time scale with the freeze-out time implies $t_{\mathrm{f}} \propto \mu^{-1 / 2}$, in agreement with our numerical results, and the classical scaling predictions $\nu=1 / 2, z=2$ for systems of classical Ginzburg-Landau type. ${ }^{15}$ It may be worth emphasizing again that this power-law scaling is a direct consequence of the stress-quench protocol and the system dynamics. In the above considerations, the quench enters through a purely local $u$-term in eqn (1), leaving the scaling law unaffected by curvature variations. For large curvatures, the amplitude equations are however expected to break down as the underlying mode approximation becomes invalid. In this regime, the curvature dependence of the differential operators in eqn (1) needs to be taken into account. Such geometric effects have been shown to affect symmetry-breaking and growth rates of curved 2D crystals. $^{52,60}$ However, since in our system the growth rate increases linearly with the quench depth at freeze-out (Appendix Amplitude equations), we hypothesize that for fast enough quenches and moderate curvature variations, the KZ-scalings remain dominant over curvature effects. Lastly, it should be noted that eqn (1) is valid only when the characteristic wavelength of the pattern, the hexagonal lattice spacing, is considerably smaller than the radius of curvature. Therefore, wrinkling on very strongly curved substrates can no longer be adequately described within the present theory.

\subsection{Nucleation dynamics in curved surface crystals}

Crystal growth in planar Euclidean geometry is well understood. By contrast, the complex interplay between kinetics, substrate curvature and defect formation is still being investigated. ${ }^{26,27}$ The current interest in these topics is in parts driven by recent technological advances in the fabrication of graphene ${ }^{65}$ and carbon nanotubes ${ }^{37}$ and by the development of modern confocal imaging techniques that make it possible to track micron-sized colloids and cells at high spatio-temporal resolution. For instance, the formation of hexagonal crystal structures similar to those described above can be observed during the early developmental stages in the fruit fly Drosophila melanogaster, when nuclei migrate to accumulate underneath the surface of the ellipsoidal shell that encapsulates the embryo. ${ }^{66}$ Similarly, ciliated somatic cells form a spherical crystal on the surface of the colonial alga Volvox carteri, ${ }^{67}$ with the cells' arrangement determining the phototactic properties of the organism.
Important insights into the kinetics of crystal growth on curved substrates were obtained recently in a joint experimental and theoretical study ${ }^{26}$ on the assembly of charged colloids on spherical liquid-liquid interfaces. The crystal formation dynamics observed in these experiments shares striking similarities with the nucleation sequences of the hexagonal wrinkling patterns shown in Fig. 1C. In both cases, one first observes the formation of several smaller highly regular crystal patches, while the defects form during the later stages when two or more of these patches merge. These kinetic parallels suggest a certain universality in the crystal formation processes on curved surfaces in the slow-quench regime. Extrapolating these similarities to higher quench rates, one may hope that the scaling results identified here translate to other physical and biological systems that develop crystalline structures on their curved surfaces.

\section{Conclusions}

The above analysis suggest that KZ-type scalings can occur in an experimentally accessible, macroscopic elastic bilayer system. Specifically, we have identified the power law scaling relation between topological defects densities and linear quench rates using a recently validated generalized Swift-Hohenberg theory of elastic bilayer wrinkling. ${ }^{49}$ Our analysis supports earlier studies which showed that $\mathrm{KZ}$ scalings can arise in the context of (weakly) first-order transitions, provided the quench rates are fast enough. $^{22,24}$ The numerical results above predict universal scalings in spherical and toroidal geometries. Further work will be needed however to test whether these results extend to other geometries with strongly varying curvature, where symmetrybreaking is expected to become inhomogeneous. With regard to applications, the KZ scaling relations for elastic surface crystals offer concrete guidance for controlling the number of topological defects by tuning the quench rate, extending previous work $^{40,41}$ that showed how substrate geometry can be used for defect localization. The nucleation sequences leading to crystalline hexagonal surface patterns in the thin-film model are qualitatively similar to those reported recently for colloidal crystals on spherical liquid-liquid interfaces. ${ }^{26}$ This suggests that elastic surface crystals, which have been realized by substrate depressurization ${ }^{46}$ or surface swelling, ${ }^{47}$ can offer a flexible testbed for exploring generic aspects of crystal growth kinetics and topological defect formation in complex geometries.

\section{Conflicts of interest}

There are no conflicts to declare.

\section{Appendix}

\section{Wrinkling theory}

Wrinkling of a thin stiff film that is adhered to a softer curved substrate can effectively be described by a Swift-Hohenberg-type equation for the normal displacement field $u{ }^{49}$ Measuring all 
length in terms of the film thickness $h$, the associated elastic energy on an arbitrary substrate geometry $\omega$ is given by, see eqn (34) and (40a) in the ESI $\dagger$ of ref. 49,

$$
\begin{aligned}
\mathscr{E}= & \frac{k}{2} \int_{\omega} \mathrm{d} \omega\left[\gamma_{0}(\nabla u)^{2}+\frac{1}{12}(\Delta u)^{2}+a u^{2}+\frac{2}{3} b u^{3}\right. \\
& \left.+\frac{c}{2} u^{4}-\Gamma(u)+\Upsilon(u)\right]
\end{aligned}
$$

with $k=E_{\mathrm{f}} /\left(1-\nu^{2}\right)$ for a film with Young's modulus $E_{\mathrm{f}}$ and Poisson ratio $\nu$. The nonlinearities are

$$
\begin{aligned}
\Gamma(u) & =\left[(1-\nu) b^{\alpha \beta} \nabla_{\alpha} u \nabla_{\beta} u+\nu b_{\gamma}^{\gamma}(\nabla u)^{2}\right] u \\
\Upsilon(u) & =\frac{1}{2}\left[(1-\nu) c^{\alpha \beta} \nabla_{\alpha} u \nabla_{\beta} u+\nu c_{\gamma}^{\gamma}(\nabla u)^{2}\right] u^{2}
\end{aligned}
$$

Here, $b_{\alpha \beta}$ is the second fundamental form (curvature tensor), and $c_{\alpha \beta}$ the third fundamental form. We note that $b=3 b_{\gamma}^{\gamma}[(3-\nu)$ $\left.\mathscr{K}-\left(b_{\gamma}^{\gamma} / 2\right)^{2}\right] / 2$, with $\mathscr{K}$ the Gaussian curvature, is of third order in the curvature tensor and thus negligible in comparison to the firstorder curvature nonlinearity $\Gamma(u)$. Moreover, numerical analysis for experimentally relevant parameters ${ }^{49}$ shows that $\Upsilon(u)$ is typically smaller than the other energy contributions and, hence, does not significantly affect surface pattern formation. We can therefore neglect the terms $b u^{3}$ and $Y(u)$ in the remainder.

Taking the variation of the remaining energy with respect to the normal displacement field $u$, and assuming overdamped dynamics, we obtain the generalized Swift-Hohenberg equation (GSHE) given in eqn (1) with ${ }^{49}$

$$
\begin{aligned}
\delta_{u} \Gamma(u)= & (\nu-1)\left[b^{\alpha \beta} \nabla_{\alpha} u \nabla_{\beta} u+2 u \nabla_{\beta}\left(b^{\alpha \beta} \nabla_{\alpha} u\right)\right] \\
& +\nu\left[b_{\gamma}^{\gamma}(\nabla u)^{2}-2 \nabla \cdot\left(b_{\gamma}^{\gamma} u \nabla u\right)\right]
\end{aligned}
$$

To first order in the substrate curvature, the various parameters in the energy and in the GSHE relate to mechanical and geometrical system properties as follows: ${ }^{49}$

$$
\begin{gathered}
\gamma_{0}=-\frac{\eta^{2 / 3}}{6}, \quad c=\frac{2(1+\nu) \eta^{2 / 3}}{3} c_{1}, \\
a=a_{\mathrm{c}}-\frac{3 c}{4} \Sigma_{\mathrm{e}}, \quad a_{\mathrm{c}}=\frac{\eta^{4 / 3}}{12}=3 \gamma_{0}^{2}
\end{gathered}
$$

with $c_{1}=0.019$ a fitting constant required to match quantitatively the wrinkling theory to experiments, and $\eta=3 E_{\mathrm{s}} / E_{\mathrm{f}}$ the ratio of Young's moduli between substrate and film, respectively. ${ }^{49}$

In our simulations, we fix the Poisson ratio $\nu=1 / 2$ and $\eta=0.33$, corresponding to a substrate that is approximately one order of magnitude softer than the adhering film, which is in the range of earlier experiments. ${ }^{48}$ To solve eqn (1) under constant quenches $\Sigma_{\mathrm{e}}=\mu t$, we use a custom subdivision surface-based finite element code with $C^{1}$-continuous basis functions. ${ }^{68,69}$ The simulations use triangular meshes consisting of up to $\sim 2.7 \times 10^{4}$ mesh nodes and $\sim 5.5 \times 10^{4}$ elements. To break the symmetry of the initially unwrinkled film $u=0$, we add a small stationary random field $\varepsilon$ to the rhs of eqn (1). The values of $\varepsilon$ at each mesh-node are drawn independently from a uniform distribution over the interval $\left[-0.5 \times 10^{-6}\right.$, $\left.0.5 \times 10^{-6}\right]$ and kept fixed throughout a simulation run.
To integrate the GSHE in time, we use a standard forward Euler time stepping integrator, noting that the dynamics is overdamped and thus numerically stable and accurate for small time steps $\Delta t$. For a given quench $\mu t$, the numerical integration is started at time $t_{\text {init }}=-10^{3} \mu \Delta t<0$, so that each simulation spends a comparable number of integration steps in the unwrinkled regime $\Sigma_{\mathrm{e}}<0$.

\section{Amplitude equations}

As outlined in ref. 49, the GSHE on spherical substrates with radius $R$ can be approximated by a standard Swift-Hohenberg (SH) equation by replacing the $\Gamma(u)$-term with a quadratic nonlinearity that produces the same average force for a pattern consisting of plane waves $\sim \mathrm{e}^{i \mathbf{k} \cdot \mathbf{x}}$ with $|\mathbf{k}|=k_{\mathrm{c}}=\sqrt{6\left|\gamma_{0}\right|}$. Keeping only the first-order curvature terms, the resulting standard $\mathrm{SH}$ equation can be written in the rescaled standard form ${ }^{49}$

$$
\partial_{T} \phi=\alpha \phi-\left(1+\Delta_{X}\right)^{2} \phi+\beta \phi^{2}-\phi^{3}
$$

where

$$
\begin{array}{cc}
T=\frac{t}{3 \gamma_{0}^{2}}, & \Delta_{X}=\frac{\Delta}{6\left|\gamma_{0}\right|} \\
\phi=\frac{u}{u_{*}}, & u_{*}=\sqrt{\frac{3}{c}\left|\gamma_{0}\right|} \\
\alpha=1-\frac{a}{a_{c}}=\frac{c}{4 \gamma_{0}^{2}} \Sigma_{\mathrm{e}}, & \beta=-\frac{2 \sqrt{3 / c}}{R}
\end{array}
$$

For non-spherical substrates, the coefficient $\beta$ becomes a function of the position on the surface $\omega$ and thus generally considerably more complex than in the spherical case. However, since an appropriate $\beta$-term can be constructed for a given surface geometry, and the value of $\beta$ is not important for the subsequent arguments, it suffices to assume in the following that the GSHE can be cast into the form (9).

We note that in the above normal form, patterns with non-zero amplitude and wave length $\lambda=2 \pi$ emerge when the bifurcation parameter $\alpha>0$ : linearizing eqn (9) with the plane-wave ansatz $u(x, t)=A \exp (\omega t+i \mathbf{k x})$, we obtain the first-order growth rates

$$
\omega=\alpha-1+2 k^{2}-k^{4}
$$

For $\alpha>0$, a band of modes around $k=1$ becomes unstable and the growth rate $\omega \sim \alpha$, i.e. unstable modes grow faster the deeper the quench $\alpha$.

Performing a standard perturbation analysis for the $\mathrm{SH}$ equation as outlined in ref. 70, we study the behavior close to bifurcation for $\alpha=B \beta^{2}$ with $|\beta| \ll 1$ and $B=O(1)$. To this end, we consider a solution to eqn (9) of the form $\phi=\beta \phi_{1}+\beta^{3} \phi_{3}+\cdots$ with ansatz

$$
\phi_{1}=\sum_{a=1}^{3}\left[A_{a}(t) \mathrm{e}^{i \mathbf{K}_{a} \cdot \mathbf{X}}+\bar{A}_{a}(t) \mathrm{e}^{-i \mathbf{K}_{a} \cdot \mathbf{X}}\right]
$$

where the wave vectors $\mathbf{K}_{1}=(1,0), \mathbf{K}_{2}=(-1 / 2, \sqrt{3} / 2)$, and $\mathbf{K}_{3}=(-1 / 2,-\sqrt{3} / 2)$. Substituting this ansatz into eqn (10) and using that $\mathbf{K}_{1}+\mathbf{K}_{2}+\mathbf{K}_{3}=0$, one finds at order $O\left(\beta^{3}\right)$ three 
amplitude equations of the form ${ }^{70}$

$$
\frac{\mathrm{d} A_{1}}{\mathrm{~d} T}=\beta^{2}\left[B A_{1}+2 \bar{A}_{2} \bar{A}_{3}-3\left|A_{1}\right|^{2} A_{1}-6\left(\left|A_{2}\right|^{2}+\left|A_{3}\right|^{2}\right) A_{1}\right]
$$

with the other two equations obtained by cyclic permutation. Splitting the three amplitude equations into six separate equations for magnitudes $R_{a}=\left|A_{a}\right|$ and complex phases $\theta_{a}=\arg \left(A_{a}\right)$, one finds $\sum_{a} \theta_{a}=0$ as an attractive fix point, meaning that phases synchronize. ${ }^{70}$ Without loss of generality, one can assume individually $\theta_{a}=0,{ }^{70}$ corresponding to a real amplitude ansatz.

Hexagons are characterized by equal amplitudes $R_{a} \equiv \bar{R}$, leading to a single amplitude equation

$$
\frac{\mathrm{d} \bar{R}}{\mathrm{~d} T}=\beta^{2}\left(B \bar{R}+2 \bar{R}^{2}-15 \bar{R}^{3}\right)
$$

To leading order, we then have in the original units $u=U(t) \sum_{a=1}^{3}\left(\mathrm{e}^{i \mathbf{k}_{a} \cdot \mathbf{x}}+\mathrm{e}^{-i \mathbf{k}_{a} \cdot \mathbf{x}}\right)$ with $\mathbf{k}_{\mathrm{a}}=k_{\mathrm{c}} \mathbf{K}_{\mathrm{a}}$ as given in Discussion and $U(t)=u_{*} \beta \bar{R}(t)$. Hence, combining eqn (10) and (13), we obtain

$$
\frac{\mathrm{d} U}{\mathrm{~d} t}=\frac{c \Sigma_{\mathrm{e}}}{12 \gamma_{0}{ }^{4}} U-\frac{4}{3 R\left|\gamma_{0}\right|^{3}} U^{2}-\frac{15 c}{9 \gamma_{0}{ }^{4}} U^{3}
$$

Close to the onset of bifurcation, $U \ll 1$ and the higher order terms $O\left(U^{2}\right)$ can be neglected, yielding eqn (4). Note that curvature-dependent terms appear only at second order in $U$, suggesting that the dynamics close to onset is insensitive to local curvature variation, in agreement with the observed KZ-scalings on toroidal geometries presented. Moreover, for spherical substrates, the stable stationary solution of eqn (14) is spatially invariant and given by

$$
|U|=\frac{\left|\gamma_{0}\right|}{10 R}\left(4+\sqrt{16+\frac{5 c R^{2} \Sigma_{\mathrm{e}}}{\gamma_{0}^{2}}}\right)
$$

with $R$ the radius of the sphere. In the adiabatic limit $\mu \rightarrow 0$, we have

$$
\left\langle u^{2}\right\rangle_{\mu \rightarrow 0} \propto U^{2}=\left(\frac{4 \gamma_{0}}{5 R}\right)^{2}+\frac{c}{10} \Sigma_{\mathrm{e}}+O\left(\Sigma_{\mathrm{e}}^{2}\right),
$$

implying a linear growth of the order parameter with $\Sigma_{\mathrm{e}}$, in agreement with Fig. 2D.

For toroidal geometries (Fig. 3C and 4A), we find a similar behavior for substrates with non-constant curvature, confirming that curvature variations do not greatly affect the surface-averaged amplitude for the considered toroidal geometries. Specifically, we estimate for toroidal geometries from Fig. 3C

$$
\left\langle u^{2}\right\rangle_{\mu \rightarrow 0}=A+B \Sigma_{\mathrm{e}}+O\left(\Sigma_{\mathrm{e}}^{2}\right)
$$

with $A \approx 0.38$ and $B \approx 0.32$ (solid black line in Fig. 4A).

\section{Freeze-out}

We summarize how the freeze-out time $t_{\mathrm{f}}$ and the corresponding stress $\Sigma_{\mathrm{e}, \mathrm{f}}$ are obtained for toroidal geometries. The procedure for spherical substrates is analogous.

We first determine the stress $\Sigma_{\max }$ and corresponding time $t_{\max }=\Sigma_{\max } / \mu$ where $\left\langle u^{2}\right\rangle$ has largest slope. We find that $\Sigma_{\max }-$ $\Sigma_{0} \propto \mu^{1 / 2}$, confirming the KZ-type scaling (Fig. 4A and B). Since amplitudes are however not yet fully developed at $t_{\max }$, we add a time delay to allow amplitudes of hexagonal patterns to fully develop. Under a constant-rate quench $\Sigma_{\mathrm{e}}=\mu t$, the amplitude eqn (14) become quench-rate independent under the rescaling $t^{\prime}=t \mu^{1 / 2}$ at leading order in $U$. Choosing the delay $\Delta \tau \mu^{-1 / 2}$ thus allows each quench the same (rescaled) time to fully develop the hexagonal amplitudes. We chose the value of $\Delta \tau=30$, which is long enough to allow fully developed hexagonal patterns, while also being small enough that defect annihilation is still negligible, thus not affecting the KZ scaling of the defect density. Accordingly, the freeze-out film stress is defined as $\Sigma_{\mathrm{e}, \mathrm{f}}=\mu t_{\mathrm{f}}=\Sigma_{\max }+\mu^{1 / 2} \Delta \tau$.

To confirm the scalings of the order parameter in the transition region near $t_{\max }$, we first shift the measured quench curves of Fig. $4 \mathrm{~A}$ by $t_{\max }$ and rescale time by $\mu^{1 / 2}$. For better comparison, we then normalize the order parameter $\left\langle u^{2}\right\rangle$ as
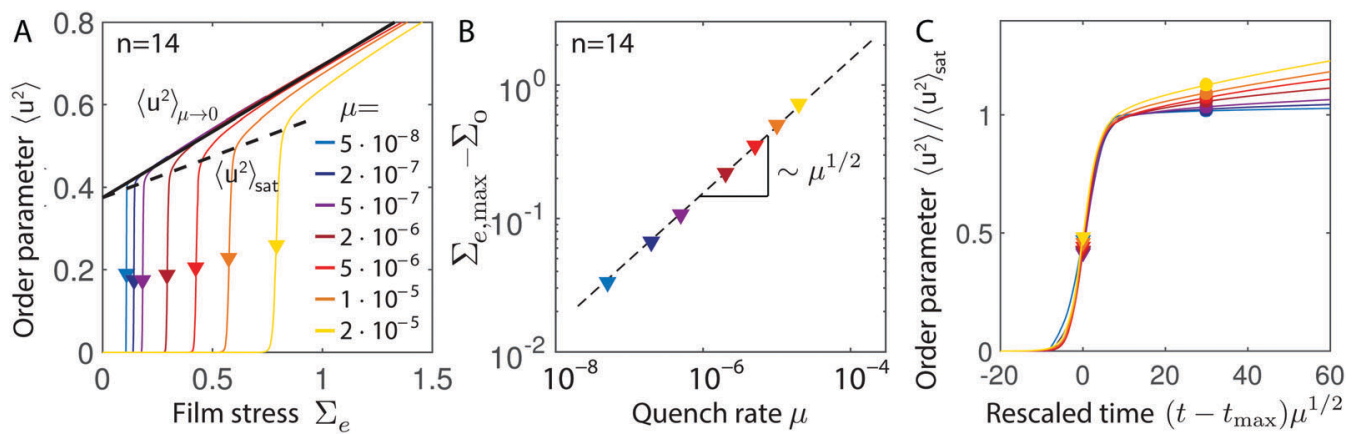

Fig. 4 Determination of freeze-out time and stress for toroidal surface crystal. (A) Delayed bifurcation of the order parameter $\left\langle u^{2}\right\rangle$, identical to Fig. $3 C$. Markers denote the location $\Sigma_{\mathrm{e} \text {,max }}$ of largest slope. The solid line is the linear approximation $\left\langle u^{2}\right\rangle_{\mu \rightarrow 0}=0.38+0.32 \Sigma_{\mathrm{e}}$, whereas the dashed line, $\left\langle u^{2}\right\rangle_{\text {sat }}=0.37+0.2 \Sigma_{\mathrm{e}}$ demarcates the region where the order parameter begins to saturate. (B) The maximum slope stresses follow the KZ scaling, $\Sigma_{\mathrm{e}, \max }-\Sigma_{0} \propto \mu^{1 / 2}$. (C) Close to $\Sigma_{\mathrm{e}, \max }$ and the corresponding time $t_{\max }$, the dynamics of the normalized order parameter $\left\langle u^{2}\right\rangle /\left\langle u^{2}\right\rangle_{\mu \rightarrow 0, \text { sat }}$ is universal under the rescaling $t \rightarrow t \mu^{1 / 2}$. Circle symbols indicate the freeze-out times $t_{\mathrm{f}}=t_{\max }+\Delta \tau \mu^{-1 / 2}(\Delta \tau=30)$. 
follows: from Fig. 4A, we observe that for all quench rates, the order parameter curves begin to saturate at values of $\left\langle u^{2}\right\rangle_{\text {sat }}$ that are linear in $\Sigma_{\mathrm{e}}$ (dashed line in Fig. 4A). Estimating the linear trend, we find $\left\langle u^{2}\right\rangle_{\text {sat }}=0.37+0.2 \Sigma_{\mathrm{e}}$. We thus normalize $\langle u\rangle$ by $\left\langle u^{2}\right\rangle_{\text {sat }}$ evaluated at $\Sigma_{\text {e,max }}$. Under these rescalings, the curves $\left\langle u^{2}\right\rangle /\left\langle u^{2}\right\rangle_{\mu \rightarrow 0 \text {,sat }}$ for various quenches $\mu$ collapse onto a single curve in the transition region close to $t=t_{\max }$ (Fig. 4C). We emphasize that it is impossible to simultaneously collapse order parameter curves after the freeze-out, because for $t \gg t_{\max }$ the order parameter asymptotes to the adiabatic value, which scales linearly in the quench rate and time, $\left\langle u^{2}\right\rangle_{\mu \rightarrow 0} \simeq$ $A+B \Sigma_{\mathrm{e}}=A+B \mu t$, explaining the different slopes observed for $t \gg t_{\text {max }}$ in Fig. 4C.

\section{Pattern analysis}

To reconstruct the curved crystal structure and detect topological defects, we first threshold the amplitude field $u$ obtained from simulations to find the center of each crystalline lattice site. Each site is then connected to its nearest neighbors via a Delaunay triangulation, and the hexagonal crystal structure as well as defects are obtained from the dual Voronoi graph. To find the flat crystal representation of Fig. 1C and E, we first construct the Voronoi cells of the crystal. We then randomly select a regular crystal site $s_{0}$ and its six neighbors as center of the planar lattice. Based on their positions relative to $s_{0}$, the neighbors can be assigned to one of the six surrounding unit lattice positions. We then repeat this construction sequentially for each neighbor by aligning their closest neighbors with the existing planar lattice structure, following the procedure used in ref. 26. To obtain the time evolution, we first construct the final planar crystal from the fully crystallized configuration at freeze-out time $t_{\mathrm{f}}$. For all earlier times $t<t_{\mathrm{f}}$, we use a Hungarian matching algorithm ${ }^{71}$ to track identical Voronoi sites and identify them in the fully crystallized planar configuration.

\section{References}

1 I. Chuang, R. Durrer, N. Turok and B. Yurke, Science, 1991, 251, 1336.

2 P.-G. De Gennes and J. Prost, The Physics of Liquid Crystals, Clarendon Press, Oxford, 1995.

3 M. Nikkhou, M. Skarabot, S. Copar, M. Ravnik, S. Zumer and I. Musevic, Nat. Phys., 2015, 11, 183-187.

4 X. Wang, D. S. Miller, E. Bukusoglu, J. J. de Pablo and N. L. Abbott, Nat. Mater., 2016, 15, 106-112.

5 P. Weiss, C. R. Acad. Sci., 1906, 143, 1136.

6 T. D. Edwards, Y. Yang, D. J. Beltran-Villegas and M. A. Bevan, Sci. Rep., 2014, 4, 6132.

7 A. Cortijo and M. A. H. Vozmediano, EPL, 2007, 77, 47002.

8 O. V. Yazyev and L. Helm, Phys. Rev. B: Condens. Matter Mater. Phys., 2007, 75, 125408.

9 W. H. Zurek, Nature, 1985, 317, 505-508.

10 P. C. Hendry, N. S. Lawson, R. A. M. Lee, P. V. E. McClintock and C. D. H. Williams, Nature, 1994, 368, 315-317.
11 M. J. W. Dodgson and M. A. Moore, Phys. Rev. B: Condens. Matter Mater. Phys., 1997, 55, 3816-3831.

12 R. Monaco, J. Mygind, R. J. Rivers and V. P. Koshelets, Phys. Rev. B: Condens. Matter Mater. Phys., 2009, 80, 180501.

13 T. W. B. Kibble, J. Phys. A: Math. Gen., 1976, 9, 1387.

14 M. Hindmarsh, J. Phys. A: Math. Theor., 2016, 49, 411001.

15 W. H. Zurek, Phys. Rep., 1996, 276, 177-221.

16 N. Fowler and I. Dierking, ChemPhysChem, 2017, 18, 812-816.

17 S. Deutschländer, P. Dillmann, G. Maret and P. Keim, Proc. Natl. Acad. Sci. U. S. A., 2015, 112, 6925-6930.

18 S. Ulm, J. Roßnagel, G. Jacob, C. Degünther, S. T. Dawkins, U. G. Poschinger, R. Nigmatullin, A. Retzker, M. B. Plenio and F. Schmidt-Kaler, et al., Nat. Commun., 2013, 4, 2290.

19 M. Anquez, B. A. Robbins, H. M. Bharath, M. Boguslawski, T. M. Hoang and M. S. Chapman, Phys. Rev. Lett., 2016, 116, 155301.

20 V. M. H. Ruutu, V. B. Eltsov, A. J. Gill, T. W. B. Kibble, M. Krusius, Y. G. Makhlin, B. Placais, G. E. Volovik and W. Xu, Nature, 1996, 382, 334-336.

21 G. Labeyrie and R. Kaiser, Phys. Rev. Lett., 2016, 117, 275701.

22 S. Casado, W. González-Viñas, H. Mancini and S. Boccaletti, Phys. Rev. E: Stat., Nonlinear, Soft Matter Phys., 2001, 63, 057301.

23 M. A. Miranda, D. Laroze and W. González-Viñas, J. Phys.: Condens. Matter, 2013, 25, 404208.

24 S. Casado, W. González-Viñas, S. Boccaletti, P. L. Ramazza and H. Mancini, Eur. Phys. J.-Spec. Top., 2007, 146, 87-98.

25 T. Galla and E. Moro, Phys. Rev. E: Stat., Nonlinear, Soft Matter Phys., 2003, 67, 035101.

26 G. Meng, J. Paulose, D. R. Nelson and V. N. Manoharan, Science, 2014, 343, 634-637.

27 D. A. Beller and D. R. Nelson, Phys. Rev. E, 2016, 94, 033004.

28 R. E. Guerra, C. P. Kelleher, A. D. Hollingsworth and P. M. Chaikin, Nature, 2018, 554, 346-350.

29 M. Pennybacker and A. C. Newell, Phys. Rev. Lett., 2013, 110, 248104.

30 J. F. Sadoc, J. Charvolin and N. Rivier, J. Phys. A: Math. Theor., 2013, 46, 295202.

31 M. F. Pennybacker, P. D. Shipman and A. C. Newell, J. Phys. D: Appl. Phys., 2015, 306, 48-81.

32 A. Amir, F. Babaeipour, D. B. McIntosh, D. R. Nelson and S. Jun, Proc. Natl. Acad. Sci. U. S. A., 2014, 111, 5778-5783.

33 A. Amir, J. Paulose and D. R. Nelson, Phys. Rev. E: Stat., Nonlinear, Soft Matter Phys., 2013, 87, 042314.

34 B. Berger, P. W. Shor, L. Tucker-Kellogg and J. King, Proc. Natl. Acad. Sci. U. S. A., 1994, 91, 7732-7736.

35 J. Lidmar, L. Mirny and D. R. Nelson, Phys. Rev. E: Stat., Nonlinear, Soft Matter Phys., 2003, 68, 051910.

36 D. Chrétien, F. Metoz, F. Verde, E. Karsenti and R. H. Wade, J. Cell Biol., 1992, 117, 1031.

37 J. A. Robinson, E. S. Snow, S. C. Badescu, T. L. Reinecke and F. K. Perkins, Nano Lett., 2006, 6, 1747-1751.

38 E. P. Chan and A. J. Crosby, Adv. Mater., 2006, 18, 3238-3242.

39 L. Euler, Novi Comment. Acad. Sci. Imp. Petropol., 1758, 4, 109-140. 
40 L. Giomi and M. J. Bowick, Phys. Rev. E: Stat., Nonlinear, Soft Matter Phys., 2008, 78, 010601.

41 J. Francisc López, N. Stoop, R. Lagrange, J. Dunkel and P. M. Reis, Phys. Rev. Lett., 2016, 116, 104301.

42 R. Backofen, A. Voigt and T. Witkowski, Phys. Rev. E: Stat., Nonlinear, Soft Matter Phys., 2010, 81, 025701.

43 V. Vitelli, J. B. Lucks and D. R. Nelson, Proc. Natl. Acad. Sci. U. S. A., 2006, 103, 12323-12328.

44 W. T. M. Irvine, V. Vitelli and P. M. Chaikin, Nature, 2010, 468, 947-951.

45 H. Kusumaatmaja and D. J. Wales, Phys. Rev. Lett., 2013, 110, 165502.

46 M. Brojan, D. Terwagne, R. Lagrange and P. M. Reis, Proc. Natl. Acad. Sci. U. S. A., 2015, 112, 14-19.

47 D. Breid and A. J. Crosby, Soft Matter, 2013, 9, 3624-3630.

48 D. Terwagne, M. Brojan and P. M. Reis, Adv. Mater., 2014, 26, 6608-6611.

49 N. Stoop, R. Lagrange, D. Terwagne, P. M. Reis and J. Dunkel, Nat. Mater., 2015, 14, 337-342.

50 P. G. Ciarlet, Mathematical elasticity, vol. III: Theory of shells, North Holland, Amsterdam, 2000.

51 S. Cai, D. Breid, A. J. Crosby, Z. Suo and J. W. Hutchinson, J. Mech. Phys. Solids, 2011, 59, 1094-1114.

52 L. R. Gómez, N. A. Garca, V. Vitelli, J. Lorenzana and D. A. Vega, Nat. Commun., 2015, 6, 6856.

53 A. D. Pezzutti, L. R. Gómez, M. A. Villar and D. A. Vega, Europhys. Lett., 2009, 87, 66003.

54 D. A. Vega and L. R. Gómez, Phys. Rev. E: Stat., Nonlinear, Soft Matter Phys., 2009, 79, 051607.

55 T. Erneux and P. Mandel, SIAM J. Appl. Math., 1986, 46, 1-15. 56 P. Mandel and T. Erneux, J. Stat. Phys., 1987, 48, 1059-1070.
57 M. J. Bowick, A. Cacciuto, D. R. Nelson and A. Travesset, Phys. Rev. Lett., 2002, 89, 185502.

58 M. J. Bowick, D. R. Nelson and A. Travesset, Phys. Rev. B: Condens. Matter Mater. Phys., 2000, 62, 8738.

59 A. R. Bausch, M. J. Bowick, A. Cacciuto, A. D. Dinsmore, M. F. Hsu, D. R. Nelson, M. G. Nikolaides, A. Travesset and D. A. Weitz, Science, 2003, 299, 1716-1718.

60 N. A. Garca, A. D. Pezzutti, R. A. Register, D. A. Vega and L. R. Gómez, Soft Matter, 2015, 11, 898-907.

61 M. J. Bowick, D. R. Nelson and A. Travesset, Phys. Rev. E: Stat., Nonlinear, Soft Matter Phys., 2004, 69, 041102.

62 L. Giomi and M. J. Bowick, Eur. Phys. J. E: Soft Matter Biol. Phys., 2008, 27, 275-296.

63 A. Doelman, B. Sandstede, A. Scheel and G. Schneider, Eur. J. Appl. Math., 2003, 14, 85-110.

64 P. Hohenberg and A. Krekhov, Phys. Rep., 2015, 572, 1-42.

65 A. Chuvilin, U. Kaiser, E. Bichoutskaia, N. A. Besley and A. N. Khlobystov, Nat. Chem., 2010, 2, 450-453.

66 R. Tomer, K. Khairy, F. Amat and P. J. Keller, Nat. Methods, 2012, 9, 755-763.

67 K. Drescher, R. E. Goldstein and I. Tuval, Proc. Natl. Acad. Sci. U. S. A., 2010, 107, 11171-11176.

68 F. Cirak, M. Ortiz and P. Schröder, Int. J. Numer. Meth. Eng., 2000, 47, 2039-2072.

69 R. Vetter, N. Stoop, T. Jenni, F. K. Wittel and H. J. Herrmann, Int. J. Numer. Meth. Eng., 2013, 95, 791-810.

70 A. A. Golovin and A. A. Nepomnyashchy, Self-assembly, pattern formation and growth phenomena in nano-systems, Springer, Dordrecht, 2006.

71 J. Munkres, J. Soc. Ind. Appl. Math., 1957, 5, 32-38. 